\title{
IMPLEMENTACIÓN DE UN SOFTWARE MODULAR EN LA CUENCA DEL ARROYO LAS TURBIAS. PROVINCIA DE SANTA FE, ARGENTINA
}

\section{IMPLEMENTATION OF A MODULAR SOFTWARE IN LAS TURBIAS STREAM WATERSHED, PROVINCE OF SANTA FE, ARGENTINE}

\author{
Javier Vergara $^{(1)(*)}$, Maria del Valle Morresi ${ }^{(1)}$ y Mario Gardiol ${ }^{(1)}$ \\ ${ }^{(1)}$ Facultad de Ingeniería y Ciencias Hídricas, Universidad Nacional del Litoral. Santa Fe, Santa Fe, Argentina \\ ${ }^{(*)}$ e-mail: javieravergara94@gmail.com
}

\begin{abstract}
RESUMEN
En los últimos 10 años, en la cuenca del arroyo Las Turbias del centro sur de la provincia de Santa Fe, se han producido crecidas más frecuentes ante menores montos precipitados. Como consecuencia, se presentan desbordes y anegamientos prolongados y en menor grado procesos erosivos puntuales en márgenes del cauce. Estos efectos de la amenaza natural se agravan por las intervenciones individuales, no planificadas, ejecución de obras viales, canales, bordos y terraplenes de defensa como también cambios tecnológicos en la producción agrícola, que modifican permanentemente la dinámica de escurrimiento. A esta situación se suman las inquietudes e incertidumbres de los productores de la zona en cuanto al trasvase de caudales desde otras cuencas. En este trabajo se implementó el software modular CAPRA GIS para representar áreas de inundación generadas por distintos escenarios de frecuencia probabilística. La simulación se realizó con el módulo ERN- Inundación en el tramo superior de la cuenca del arroyo Las Turbias para hietogramas de diseño de diferentes recurrencias. Los mapas obtenidos sirven de apoyo a los tomadores de decisión para que junto con los organismos de cuenca gestionen de forma adecuada las medidas estructurales y no estructurales correspondientes.
\end{abstract}

Palabras clave: hidrología, hidráulica, inundación, riesgo, amenaza

\begin{abstract}
In the last 10 years, in Las Turbias stream basin placed in the south center of Santa Fe province, more frequent floods have been ocurried despite to lower precipitated amounts. As a consequence, there are prolonged overflows and floodings, and to a lesser degree erosive processes on the river banks. These effects of the natural threat are aggravated by individual and unplanned actions, execution of road works, channels and defense embankments as well as technological changes in agricultural production, which permanently modify the runoff dynamics. To this situation are added the concerns and uncertainties of the producers about flows transfer from other basins. In this work, the CAPRA GIS modular software was implemented to represent flood areas generated by different probabilistic frequency scenarios. The simulation was performed with the ERN- Inundación module in the upper section of the Las Turbias stream basin for design hyetograms of different recurrences. The maps obtained are support decision-makers so that together with the basin organizations they adequately manage the corresponding structural and non-structural measures.
\end{abstract}

Keywords: hydrology, hydraulics, flood, risk, threat 


\section{INTRODUCCIÓN}

El arroyo Las Turbias es un afluente del arroyo Carrizales-Monje, tiene una longitud de $81 \mathrm{~km}$ y la cuenca hidrográfica ocupa una superficie aproximada de $1000 \mathrm{~km}^{2}$. Abarca los departamentos Belgrano (distritos, Las Rosas y Bouquet), Iriondo (distrito Clason), San Jerónimo (distritos San Genaro y Díaz) y San Martín (distritos María Susana y Los Cardos), entre $61^{\circ} 55^{\prime} 48^{\prime}$ " y $61^{\circ} 09^{\prime} 54^{\prime \prime}$ de longitud oeste y $32^{\circ} 27^{\prime} 18^{\prime \prime}$ y $32^{\circ} 16^{\prime} 00^{\prime}$ ' de latitud sur, como muestra la Figura 1. En su extremo aguas arriba, cuenca alta- el arroyo recibe las aguas de una importante red de canales y bajos naturales (paleocañadas) correspondientes principalmente a los distritos de María Susana, Bouquet y Las Rosas. Esta zona se caracteriza, además, por su escasa pendiente (menor al 1\%) y la presencia de una amplia red vial que juegan un rol muy importante en el sistema de drenaje. La zona media de la cuenca, (comprendida entre las rutas provincial $\mathrm{N}^{\circ} 13 \mathrm{y}$ nacional $\mathrm{N}^{\mathrm{o}} 34$ aproximadamente), por el contrario, tiene pendientes importantes, que llegan en algunas zonas al $3 \%$ y no posee los rasgos fisiográficos mencionados para la alta cuenca, por lo que no presenta problemas de anegamiento. La cuenca inferior, comprendida entre la ruta nacional $\mathrm{N}^{\circ} 34 \mathrm{y}$ la desembocadura en el arroyo Carrizales-Monje, es también una zona con bajas pendientes, aunque no inferiores a las de la alta cuenca (Morresi y Gardiol, 2017).

\section{OBJETIVO}

El objetivo del trabajo, es analizar las amenazas por precipitación e inundación, mediante el módulo ERN-Inundación del Software CAPRA-GIS, (Banco Mundial, 2009) complementado con los softwares HEC-RAS 4.1, HEC-HMS 4.2, (U.S. Army Corps of Enginners Civils. Hydrologic Engineering Center, 2010) y Civil.CAD 2017.

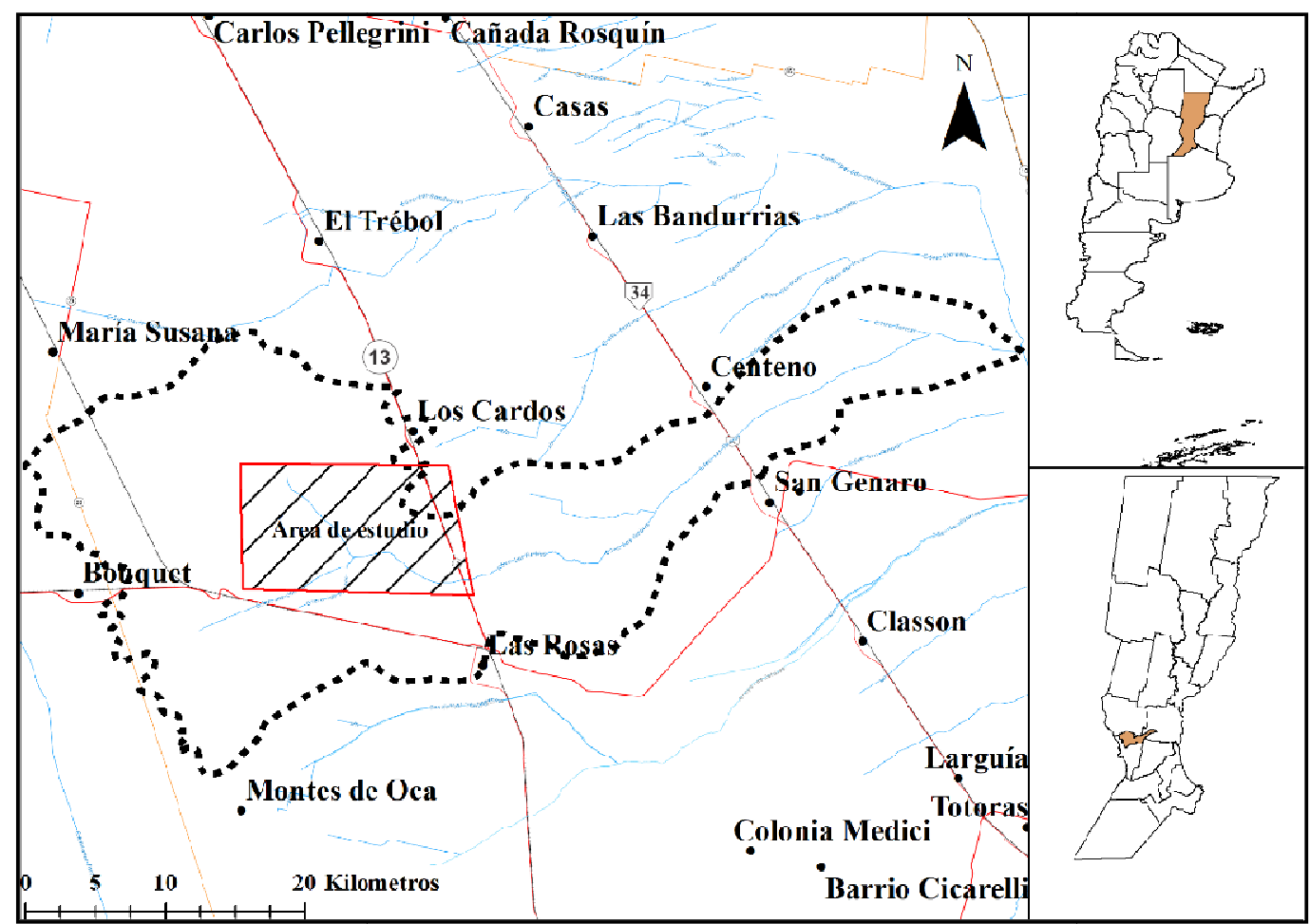

Figura 1. Cuenca arroyo Las Turbias.

\section{METODOLOGÍA}

\section{Plataforma CAPRA}

La plataforma CAPRA tiene como objetivo fortalecer la capacidad institucional para evaluar, comprender y comunicar el riesgo de desastres, con el objetivo final de integrar la información en las políticas y programas de desarrollo. Esta iniciativa comenzó en 2008, como una asociación entre el Centro de Coordinación de Prevención de Desastres Naturales en América Central (CEPREDENAC), la 
Estrategia Internacional de las Naciones Unidas para la Reducción de Desastres (UN ISDR), el Banco Interamericano de Desarrollo (BID) y El Banco Mundial como un medio para crear conciencia entre los países de América Central al proporcionarles un conjunto de herramientas que les permitirían comprender mejor el riesgo de eventos naturales adversos (Banco Mundial, 2009). Posee diversos módulos para evaluar las amenazas. Entre ellos el ER-Inundación (o ER-Flood) que permite el análisis de inundaciones de ríos, basado en un conjunto de escenarios estocásticos.

\section{Superficie TIN}

El módulo ERN-Inundación utiliza el modelo hidráulico HEC-RAS (Brunner, 2016), con perfiles transversales georreferenciados. Para ello, en el software CAD se ubicaron los perfiles transversales ortogonalmente a la línea de vaguada y se obtuvieron las coordenadas de los extremos en el marco de referencia POSGAR 2007 faja 5, correspondiente al área de estudio. Mediante las ecuaciones (1), (2) y (3) se obtuvieron el ángulo de inclinación con el norte geográfico y las coordenadas de los puntos intermedios, conocidas las de los extremos:

$$
\begin{aligned}
& \alpha=\tan ^{-1} \frac{\Delta y}{\Delta x} \\
& X_{i+1}=X_{i}+d \cdot \cos \alpha \\
& Y_{i+1}=Y_{i}+d \cdot \sin \alpha
\end{aligned}
$$

donde $\alpha$ : es el ángulo de inclinación del perfil transversal (en grados); $\Delta \mathrm{y} y \Delta \mathrm{x}$, es el incremento de las coordenadas este y norte respectivamente de los extremos del mismo; $\mathrm{X}_{\mathrm{i}} \mathrm{y} \mathrm{X}_{\mathrm{i}+1}$ coordenadas norte anterior y siguiente respectivamente; $\mathrm{Y}_{\mathrm{i}}$ y $\mathrm{Y}_{\mathrm{i}+1}$ coordenadas este anterior y siguiente respectivamente. Las coordenadas calculadas se las incorporaron al software Civil CAD, con su altimetría en cota IGN; además se consideraron las curvas de nivel de la zona para poder mejorar la definición en la zona del valle de inundación del arroyo. Luego se construyó la superficie 3D, con el método de interpolación de la red de triangulación irregular (TIN), que permitirá obtener perfiles transversales georreferenciados para poder exportar al modelo hidráulico HEC-RAS. Por último, a estos perfiles se los reproyectó al sistema de coordenadas Universal Transversal de Mercator (UTM) zona 20 sur, según lo requiere el módulo ERNINUNDACION, del software CAPRA GIS.

\section{Discretización del sistema}

Debido a que las hipótesis que supone el software de flujo se limitan a permanente, gradualmente variado, las secciones pueden ser del tipo irregular y no considera aportes laterales. Para implementar el software CAPRA en el arroyo Las Turbias se seleccionó el tramo correspondiente a la cuenca superior, progresivas $47485 \mathrm{~m}$ a $68626 \mathrm{~m}$ desde aguas abajo (aa) hacia aguas arriba (AA). El mismo se subdividió en 8 subtramos de propagación coincidentes con los aportes laterales de las subcuencas, según las siguientes progresivas (desde AA hacia aa): $68626 \mathrm{~m}$ a $65850 \mathrm{~m} ; 65850 \mathrm{~m}$ a $63850 \mathrm{~m} ; 63850 \mathrm{~m}$ a $62250 \mathrm{~m} ; 62250 \mathrm{~m}$ a $59850 \mathrm{~m}$; $59850 \mathrm{~m}$ a $57850 \mathrm{~m} ; 57850 \mathrm{~m}$ a $56171,6 \mathrm{~m} ; 56171,6$ $\mathrm{m}$ a $50250 \mathrm{~m}$ y $50250 \mathrm{~m}$ a $47485 \mathrm{~m}$. Los perfiles georreferenciados tienen asignada la progresiva propia correspondiente a la progresiva de inicio de cada subtramo considerado.

\section{Modelación hidráulica}

El módulo ERN-Inundación está acoplado al software HEC-RAS 4.1, y según las hipótesis previamente mencionadas, se resuelven las siguientes ecuaciones de continuidad (4) y de energía (5) para obtener las áreas de inundación:

$$
V_{2} \cdot A_{2}=V_{1} \cdot A_{1}
$$

$Z_{2}+Y_{2}+\frac{\alpha_{2} \cdot v_{2}^{2}}{2 g}=Z_{1}+Y_{1}+\frac{\alpha_{1} \cdot v_{1}^{2}}{2 g}+h_{e}$

A su vez la velocidad media del flujo se obtiene mediante la ecuación de resistencia de Manning (6), Chow (1994):

$\bar{V}=\frac{1}{n} \cdot R^{\frac{2}{3}} \cdot S_{f}^{\frac{1}{2}}$

donde $Z_{1}, Z_{2}$ representan la elevación del fondo del canal aa y AA, respectivamente $(\mathrm{m}) ; \mathrm{Y}_{1}, \mathrm{Y}_{2}$, la elevación del pelo de agua $(\mathrm{m}) ; \mathrm{V}_{1}, \mathrm{~V}_{2}$, la velocidad del escurrimiento en las distintas secciones $(\mathrm{m} / \mathrm{s}) ; \bar{V}$ la velocidad media del escurrimiento $(\mathrm{m} / \mathrm{s}) ; \mathrm{A}_{1}, \mathrm{~A}_{2}$ el área mojada de las secciones transversales $\left(\mathrm{m}^{2}\right) ; \alpha_{1}$, $\alpha_{2}$ coeficiente de ponderación de velocidad, $h_{e}$ perdida de carga (m); n coeficiente de resistencia al 
escurrimiento de Manning $\left(\mathrm{s} / \mathrm{m}^{1 / 3}\right) ; \mathrm{R}$ radio hidráulico $(\mathrm{m}) ; \mathrm{S}_{\mathrm{f}}$ pendiente de energía $(\mathrm{m} / \mathrm{m})$.

Las condiciones de borde AA de cada subtramo son los hidrogramas de entrada obtenidos por modelación hidrológica con HEC-HMS a los que se les adiciona los hidrogramas de aporte lateral en las progresivas que corresponda. La condición de borde aa es la pendiente media de cada subtramo. Para tormentas reales fue posible calibrar los parámetros de la modelación hidrológica-hidráulica de la cuenca del arroyo Las Turbias (Morresi et al., 2018).

\section{Modelación hidrológica}

La modelación hidrológica se realizó con el HECHMS que tuvo en cuenta la subdivisión en subcuencas de manera de obtener los hidrogramas de entrada a cada subtramo y los laterales, requeridos para la modelación hidráulica de los subtramos, como se muestra en la Figura 2 (Morresi et al., 2018).

Los datos de entrada al modelo hidrológico son los hietogramas de diseño para cada recurrencia. De esta forma se pueden obtener los hidrogramas condiciones de borde AA del modelo hidráulico de propagación de los subtramos en cada progresiva.

El módulo ERN-Inundación necesita como dato de entrada una curva caudal $(\mathrm{Q})$ - recurrencia $(\mathrm{Tr})$ para calcular el área inundada. Los caudales máximos se obtuvieron de los hidrogramas generados por el modelo hidrológico para cada subtramo con las tormentas de diseño seleccionadas.

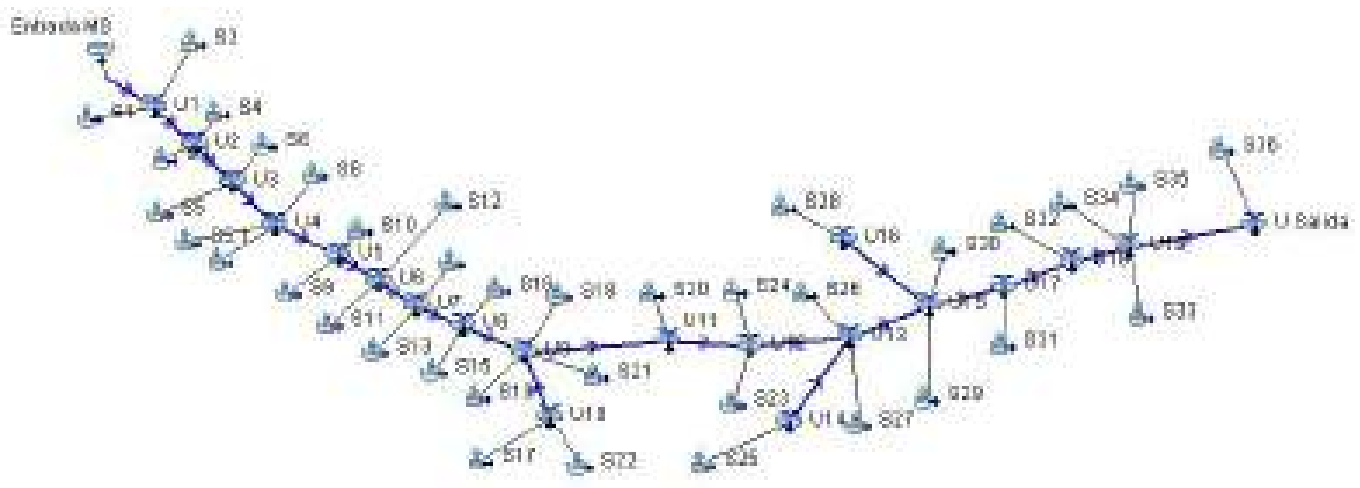

Figura 2. Esquema topológico HEC - HMS.

\section{Tormentas de diseño}

De acuerdo al tiempo de concentración de la cuenca se adoptó una duración de 5 días para las tormentas de diseño y para las recurrencias o tiempos de retorno de $2,5,10,25,50$ y 100 años.

Para construir la tormenta de diseño se utilizaron las curvas IDF de Paraná calculadas con la ecuación (7) y Figura 3.

$i=\frac{601 \cdot(T r)^{0.23}}{(d+6)^{0.69}}$

donde $\mathrm{i}$ es la intensidad $(\mathrm{mm} / \mathrm{h})$, Tr es el tiempo de retorno o recurrencia (años) y d la duración (minutos).

Según Zamanillo et al. (2008) en el patrón temporal de la precipitación para largos periodos de tiempo la mayor tasa de precipitación se produce a $1 / 3$ del tiempo total y además se aplicó un coeficiente de abatimiento areal con la ecuación (8) (Genta y Chabonnier, 2003) para suponer la uniformidad de la precipitación en la cuenca:

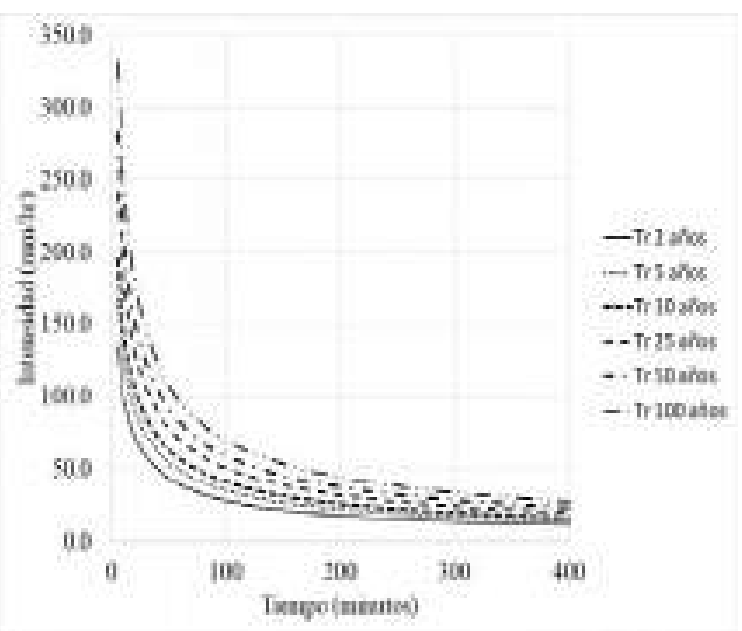

Figura 3. Curva IDF Paraná. 
$C A=1-\left(0.3549 \cdot d^{0.1272}\right)\left(1-e^{-0.005792 \cdot A}\right)$

donde: d: duración de la lluvia (horas), A área de la cuenca $\left(\mathrm{km}^{2}\right)$

La Figura 4 muestra el hietograma de diseño para una recurrencia de 2 años.

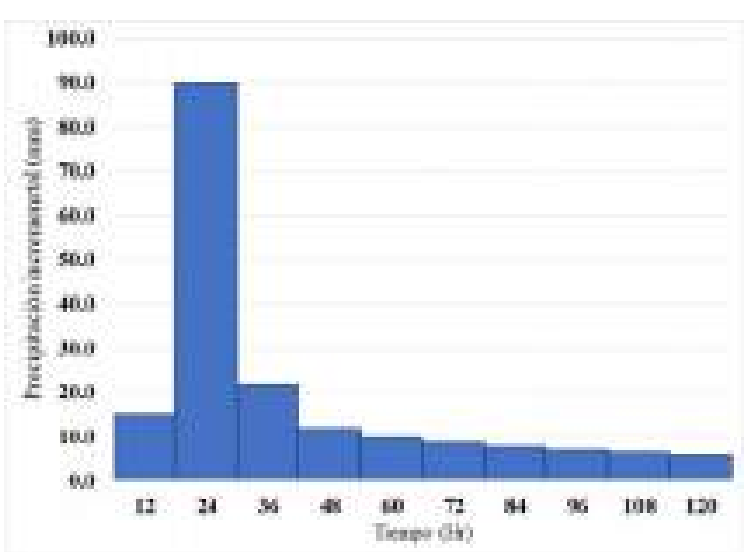

Figura 4. Tormenta de diseño $\operatorname{Tr}=2$ años.

Una vez construidas las tormentas de diseño para cada recurrencia se ingresaron como dato de entrada al modelo hidrológico de la cuenca del arroyo Las Turbias para obtener los caudales máximos (de los hidrogramas) en las secciones AA y aa de los subtramos, correspondientes a la recurrencia de la tormenta, obteniéndose la curva Q-Tr para cada subtramo.

La Figura 5 muestra la curva Q-Tr para el subtramo comprendido entre las progresivas $68626 \mathrm{~m}$ a $65850 \mathrm{~m}$.

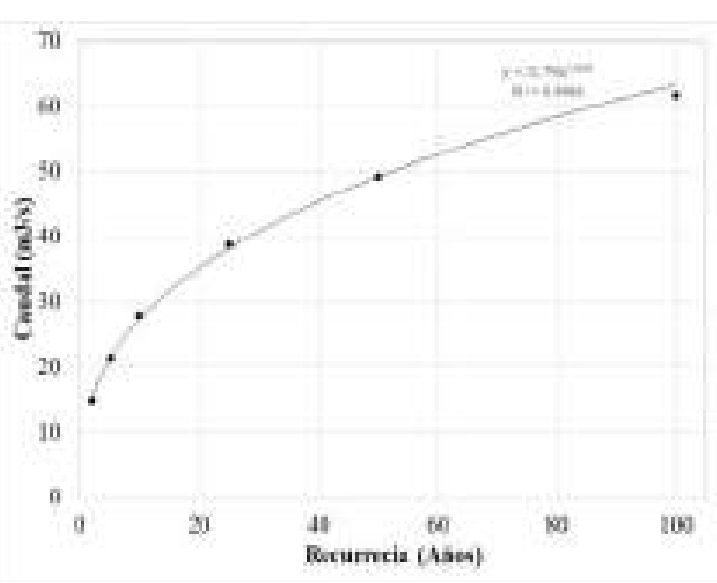

Figura 5. Curva caudal - Tr progresiva 68626 a $65850 \mathrm{~m}$.

A continuación, se presenta la Tabla 1 con los caudales del tramo superior y las recurrencias adoptadas.

\section{Implementación del módulo ERN-Inundación}

Para implementar este módulo de inundación se ingresan los perfiles georreferenciados del modelo hidráulico y las curvas caudal-recurrencia. La simulación genera diferentes escenarios en función de la recurrencia.

Las Figuras 6 y 7 muestran a modo de ejemplo los resultados del Módulo ERN-Inundación de profundidades de agua y velocidades medias del escurrimiento en el cauce y la planicie de inundación para el subtramo $68626 \mathrm{~m}$ a $65850 \mathrm{~m}$ y en la Tabla 2, se presentan las características hidráulicas $(\mathrm{Tr}=21$ años).

Tabla 1. Caudales - Recurrencia.

\begin{tabular}{|c|c|c|c|c|c|c|c|c|c|}
\hline $\begin{array}{c}\text { Progresiva } \\
(\mathrm{m})\end{array}$ & $\begin{array}{c}68626 \mathrm{a} \\
65850\end{array}$ & $\begin{array}{c}65850 \mathrm{a} \\
63850\end{array}$ & $\begin{array}{c}63850 \mathrm{a} \\
62250\end{array}$ & $\begin{array}{c}62250 \mathrm{a} \\
59850\end{array}$ & $\begin{array}{c}59850 \mathrm{a} \\
57850\end{array}$ & $\begin{array}{c}59850 \mathrm{a} \\
56191.6\end{array}$ & $\begin{array}{c}56191.6 \mathrm{a} \\
50250\end{array}$ & $\begin{array}{c}50250 \mathrm{a} \\
47485 \\
(\mathrm{RP} 13)\end{array}$ \\
\hline $\operatorname{Tr}(\mathrm{años})$ & \multicolumn{7}{|c|}{$\mathrm{Q}\left(\mathrm{m}^{3} / \mathrm{s}\right)$} \\
\hline 2 & 14.8 & 34.5 & 58.1 & 62.1 & 65.4 & 73.7 & 81.1 & 96.9 \\
\hline 5 & 21.4 & 41.2 & 64.7 & 68.7 & 74.2 & 87.7 & 98.4 & 121.4 \\
\hline 10 & 27.9 & 66.2 & 110.9 & 124.3 & 132.5 & 153.1 & 170 & 204.9 \\
\hline 25 & 38.8 & 93.2 & 156.2 & 178.4 & 190.4 & 223.4 & 248.4 & 300.4 \\
\hline 50 & 49.3 & 119.3 & 199.8 & 231.5 & 247.4 & 292.3 & 325.3 & 394.7 \\
\hline 100 & 61.8 & 150.8 & 252.9 & 296.7 & 317.1 & 376.6 & 419.2 & 509.6 \\
\hline
\end{tabular}


Se combinan los rasters generados de las distintas discretizaciones, en el software ArcGIS (ESRI, 2014) y se obtienen los mapas de afectación hídrica (Figura 8) y mapa de velocidades medias (Figura 9), con resolución de la imagen de $10 \mathrm{~m} \times 10 \mathrm{~m}$, permitiendo tener una visualización espacial de la crecida.
Se puede observar que, las profundidades máximas y las máximas velocidades, se encuentran en la zonas cercanas a las vías del ferrocarril, donde termina la simulación con un rango de profundidades de $2.5 \mathrm{~m}$ a $3 \mathrm{~m}$ y velocidades medias de $0.46 \mathrm{~m} / \mathrm{s}$ a $0.68 \mathrm{~m} / \mathrm{s}$.

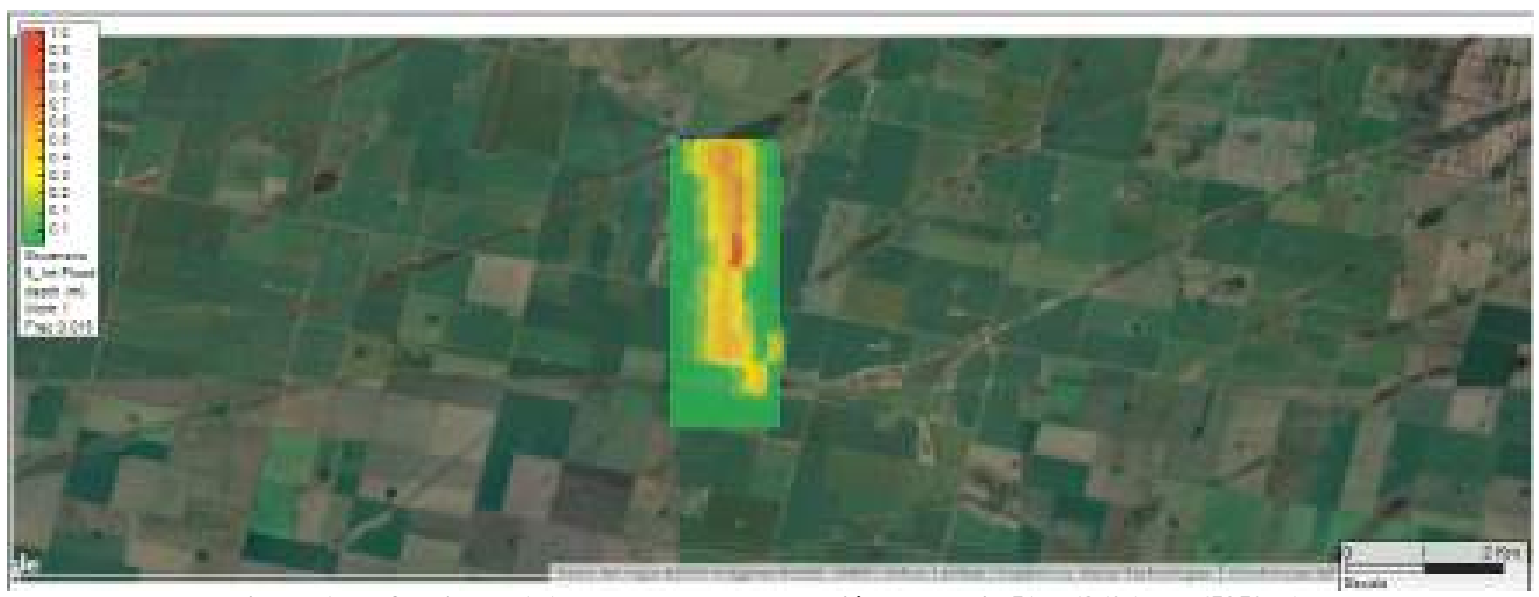

Figura 6. Profundidades(m). Modulo ERN-Inundación Escenario 5(de 68626 m a 65850 m).

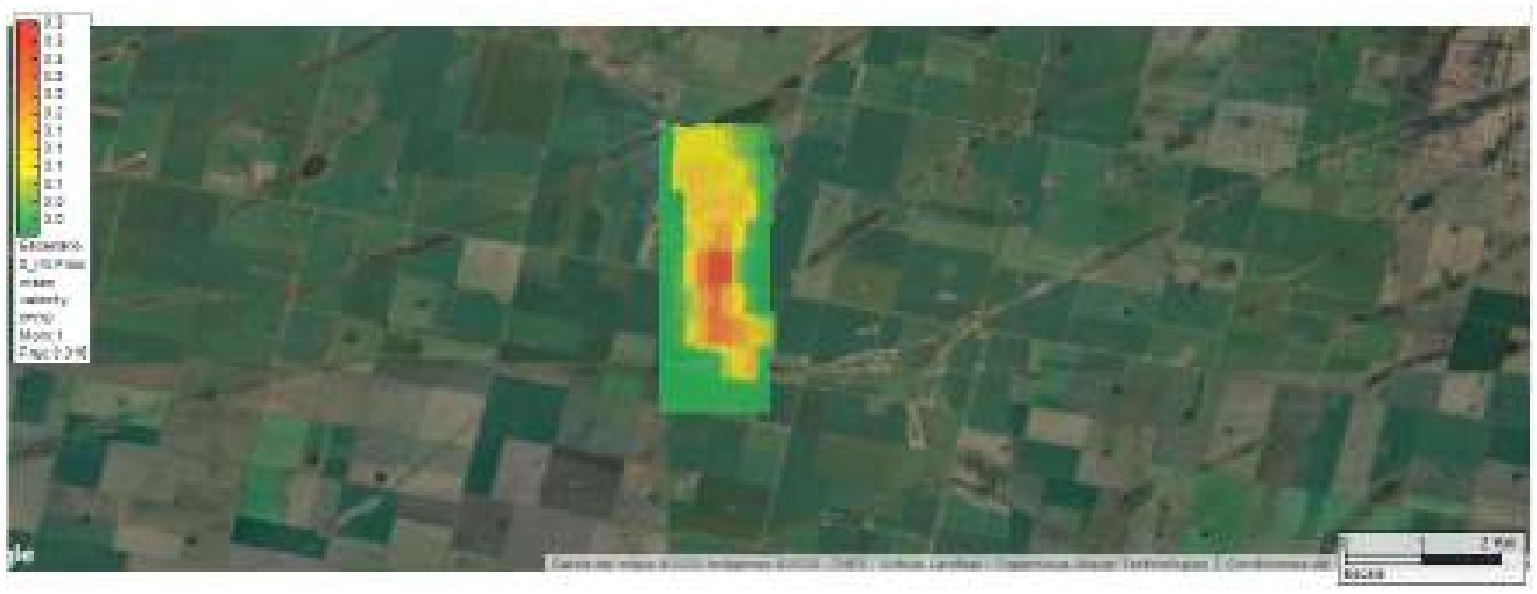

Figura 7. Velocidades medias (m/s).Modulo ERN-Inundación Escenario 5 (de 68626 m a 65850 m). 
Implementación de un software modular en la cuenca del arroyo Las Turbias. Provincia de Santa Fe, Argentina.

Tabla 2. Características Hidráulicas Subtramo $68626 \mathrm{~m}$ a $65850 \mathrm{~m}$. Perfiles georreferenciados. Tr: 21 años.

\begin{tabular}{|c|c|c|c|c|c|}
\hline Perfil & $\begin{array}{c}\text { Q Total } \\
(\mathrm{m} 3 / \mathrm{s})\end{array}$ & $\begin{array}{c}\text { Elev PA } \\
(\mathrm{m})\end{array}$ & $\begin{array}{c}\text { Vel Canal } \\
(\mathrm{m} / \mathrm{s})\end{array}$ & $\begin{array}{c}\text { Ancho Sup. } \\
(\mathrm{m})\end{array}$ & $\begin{array}{c}\text { Prof maxima } \\
(\mathrm{m})\end{array}$ \\
\hline 3461.69 & 35.95 & 102.07 & 0.16 & 1000 & 1.19 \\
\hline 3276.36 & 35.95 & 102.06 & 0.24 & 979.27 & 1.86 \\
\hline 3185.46 & 35.95 & 102.05 & 0.29 & 979.43 & 1.9 \\
\hline 3090.47 & 35.95 & 102.04 & 0.37 & 941.97 & 1.94 \\
\hline 3000 & 35.95 & 102.03 & 0.4 & 902.83 & 1.97 \\
\hline 2904.05 & 35.95 & 102.02 & 0.39 & 895.27 & 2.01 \\
\hline 2800 & 35.95 & 102.01 & 0.39 & 829.92 & 2.05 \\
\hline 2716.11 & 35.95 & 102 & 0.4 & 832.31 & 2.09 \\
\hline 2627.53 & 35.95 & 101.99 & 0.41 & 781.49 & 2.12 \\
\hline 2527.69 & 35.95 & 101.97 & 0.38 & 770.95 & 2.15 \\
\hline 2439.36 & 35.95 & 101.97 & 0.39 & 744.04 & 2.2 \\
\hline 2286.81 & 35.95 & 101.95 & 0.38 & 721.19 & 1.57 \\
\hline 2180.07 & 35.95 & 101.93 & 0.44 & 729.51 & 2.14 \\
\hline 2100 & 35.95 & 101.92 & 0.45 & 674.71 & 1.66 \\
\hline 2041.24 & 35.95 & 101.9 & 0.46 & 674.84 & 1.5 \\
\hline 1932.44 & 35.95 & 101.88 & 0.55 & 664.73 & 2.15 \\
\hline 1839.39 & 35.95 & 101.85 & 0.61 & 628.7 & 1.62 \\
\hline 1744.99 & 35.95 & 101.8 & 0.75 & 556.47 & 1.61 \\
\hline 1655.51 & 35.95 & 101.73 & 0.93 & 486.91 & 2.05 \\
\hline 1547.06 & 35.95 & 101.63 & 1.03 & 419.99 & 1.32 \\
\hline 1465.08 & 35.95 & 101.52 & 1.08 & 401.86 & 1.92 \\
\hline 1341.78 & 35.95 & 101.39 & 1.06 & 389.32 & 1.87 \\
\hline 1275.49 & 35.95 & 101.33 & 1.03 & 396.34 & 1.84 \\
\hline 1149.81 & 35.95 & 101.2 & 1.06 & 393.06 & 1.78 \\
\hline 1086.31 & 35.95 & 101.16 & 0.81 & 446.44 & 1.77 \\
\hline 947.73 & 35.95 & 101.08 & 0.72 & 515.19 & 1.74 \\
\hline 859.62 & 35.95 & 101.04 & 0.71 & 487.98 & 1.74 \\
\hline 743.98 & 35.95 & 100.97 & 0.76 & 459.4 & 1.73 \\
\hline 637.68 & 35.95 & 100.91 & 0.8 & 425.66 & 1.72 \\
\hline 549.77 & 35.95 & 100.86 & 0.77 & 437.84 & 1.72 \\
\hline 400 & 35.95 & 100.79 & 0.55 & 419.59 & 1.04 \\
\hline 277.16 & 35.95 & 100.77 & 0.5 & 456.49 & 1.81 \\
\hline 149.76 & 35.95 & 100.73 & 0.62 & 377.72 & 1.7 \\
\hline 0 & 35.95 & 100.66 & 0.68 & 320.39 & 1.46 \\
\hline
\end{tabular}




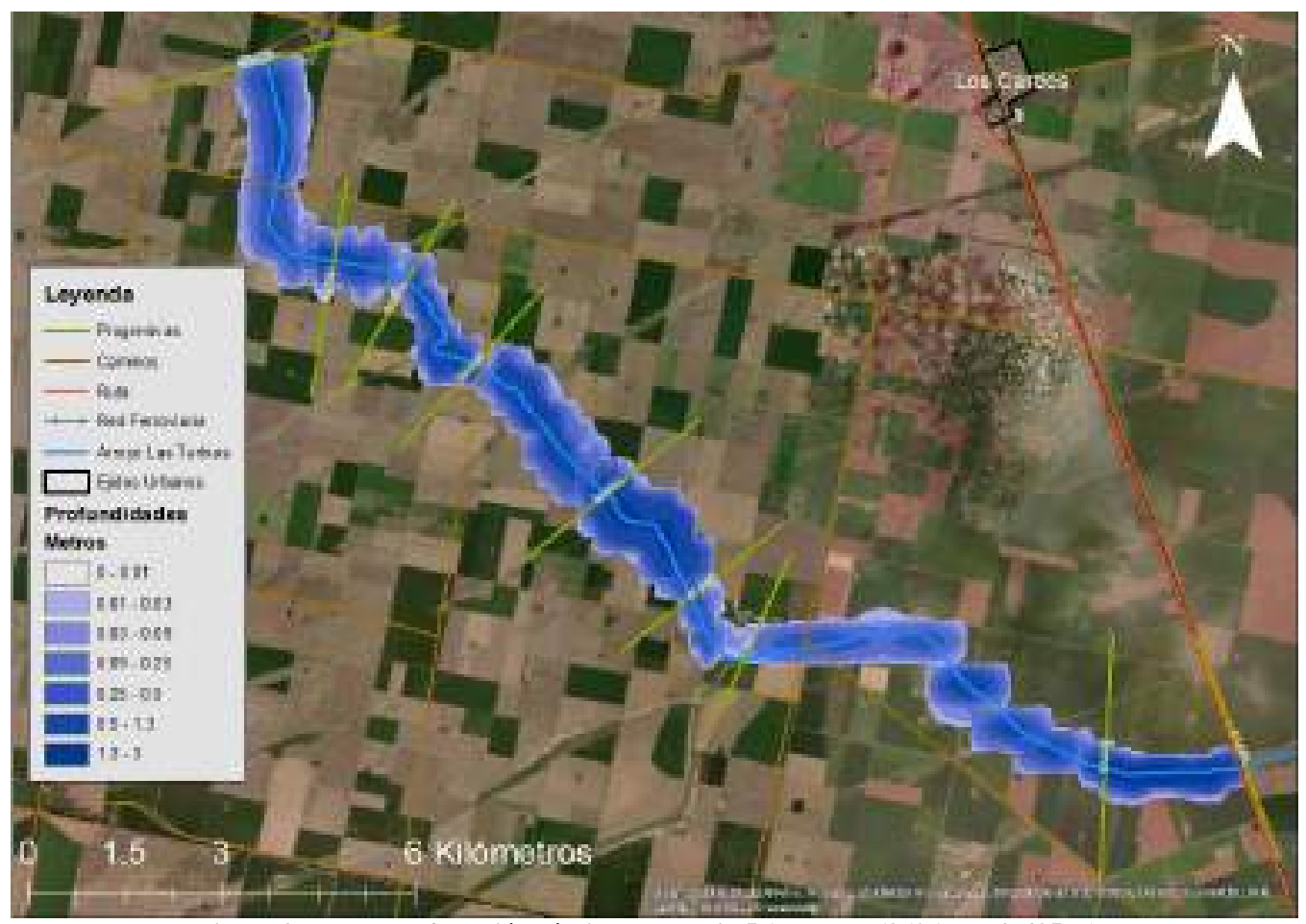

Figura 8. Mapa de afectación hídrica Escenario 5 (de prog. 68626 m a 47485 m).

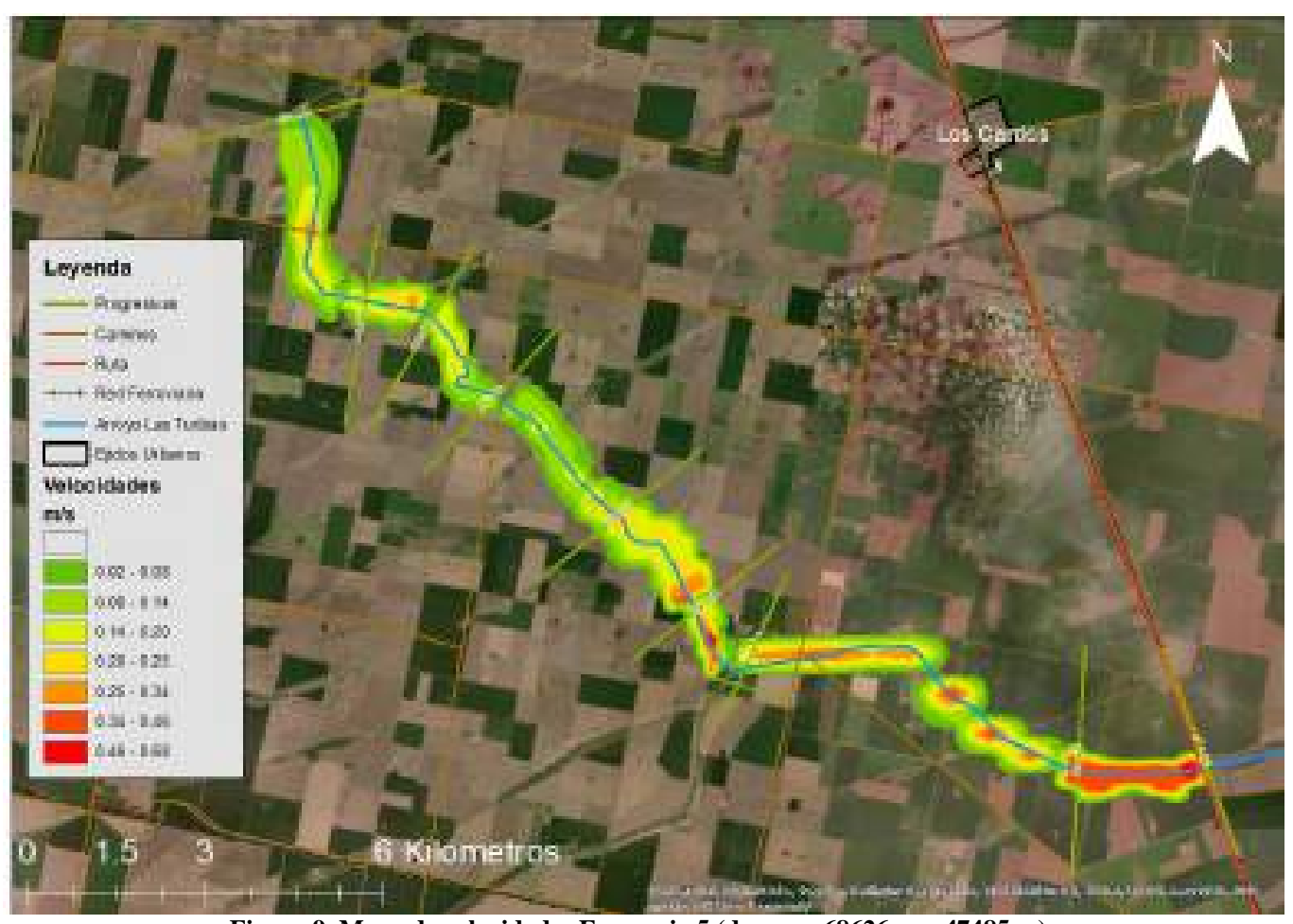

Figura 9. Mapa de velocidades Escenario 5 (de prog. 68626 m a 47485 m). 


\section{CONCLUSIONES}

El módulo ERN-Inundación del software CAPRA permite una visualización espacial a partir del mapeo de áreas de inundación.

Brinda información cuantitativa técnica de las variables hidráulicas del tramo estudiado.

Además, facilita la interpretación y análisis de los problemas de origen hídrico ocasionados por excesos de agua en la cuenca.

Esta herramienta puede contribuir a la toma de decisiones vinculadas con la planificación rural de la zona y adoptar medidas estructurales y no estructurales relacionadas a la reducción del daño debido a estos fenómenos. Además, permite vincular las amenazas por precipitación y por inundación con la frecuencia de ocurrencia del fenómeno del anegamiento.

\section{REFERENCIAS}

Banco Mundial. (2009). Manual del Usuario CAPRA-GIS. Colombia. Recuperado de https://ecapra.org/es

Brunner, G. (2016). HEC-RAS, River Analysis System Hydraulic. Davis: US. Army Corps..

Chow, V. (1994). Hidráulica de canales abiertos. Bogota: McGraw-Hill.

ESRI. (2014). Manual del usuario ArcGIS 10.2.2. Estados Unidos.

Genta, J. L. y Chabonnier, F. (2003). Curso de Hidrología Aplicada. Montevideo: Universidad de la República, Facultad de Ingeniería.

Morresi, M. y Gardiol, M. (2017). Participación de actores sociales en la cuenca del arroyo Las
Turbias. XXVI Congreso Nacional Del Agua 2017, 20 al 23 de septiembre de 2017. Córdoba, Argentina.

Morresi, M., Marcus, R., Gardiol, M. y Biancotti, E. (2018). Modelación hidrológica hidráulica con información de actores sociales en la cuenca del arroyo Las Turbias. Santa Fe. Argentina. Revista del Programa Hidrológico Internacional para América Latina y el Caribe. AQUA-LAC. Unesco. ISSN 1688-2873. En prensa.

U.S. Army Corps of Enginners Civils. Hydrologic Engineering Center. (2010) Hydrologic Modeling Center HEC-HMS User's Manual. Davis, California.

Zamanillo, E., Larenze, G., Tito, M. J., Perez, M. y Garat, M. E. (2008). Manual de Tormentas de diseño para la Provincia de Entre Rios. Buenos Aires: Universidad Tecnológica Nacional.

\section{Tipo de Publicación: NOTA TÉCNICA.}

Trabajo recibido el 27/12/2019 y aprobado para su publicación el 10/07/2020.

\section{COMO CITAR}

Vergara, J.; Morresi, M. y Gardiol, M. (2020). Implementación de un software modular en la cuenca del arroyo Las Turbias. Provincia de Santa Fe, Argentina. Cuadernos del CURIHAM. 26: 31-39. DOI: https://doi.org/10.35305/curiham.v26i0.144

Este es un artículo de acceso abierto bajo licencia: Creative Commons Atribución - No Comercial Compartir Igual 4.0 Internacional (CC BY-NC-SA 4.0) (https://creativecommons.org/licenses/by-ncsa/4.0/deed.es) 